TAIWANESE JOURNAL OF MATHEMATICS

Vol. 17, No. 2, pp. 629-659, April 2013

DOI: $10.11650 /$ tjm.17.2013.2191

This paper is available online at http://journal.taiwanmathsoc.org.tw

\title{
RIEMANNIAN SUBMERSIONS FROM ALMOST HERMITIAN MANIFOLDS
}

\author{
Bayram Şahin
}

\begin{abstract}
We survey main results of holomorphic submersions, anti-invariant submersions, slant submersions, semi-invariant submersions and semi-slant submersions defined on almost Hermitian manifolds. We also give an application of Riemannian submersions on redundant robotic chains obtained by Altafini and propose some open problems related to topics discussed in the paper.
\end{abstract}

\section{INTRODUCTION}

Riemannian submersions between Riemannian manifolds were studied by O'Neill [24] and Gray [17]. Later such submersions were considered between manifolds endowed with differentiable structures. As an analogue of holomorphic submanifolds, Watson defined almost Hermitian submersions between almost Hermitian manifolds and he showed that the base manifold and each fiber have the same kind of structure as the total space, in most cases [37]. We note that almost Hermitian submersions have been extended to the almost contact manifolds [12], locally conformal Kähler manifolds[22] and quaternion Kähler manifolds [20] (see:[15] for details concerning Riemannian submersions between Riemannian manifolds equipped with additional structures of complex, contact, locally conformal or quaternion Kähler).

In [32], we introduced anti-invariant Riemannian submersions from almost Hermitian manifolds onto Riemannian manifolds as follows. Let $M$ be a complex $m-$ dimensional almost Hermitian manifold with Hermitian metric $g_{M}$ and almost complex structure $J$ and $N$ be a Riemannian manifold with Riemannian metric $g_{N}$. Suppose that there exists a Riemannian submersion $F: M \longrightarrow N$ such that the integral manifold of the distribution $k e r F_{*}$ is anti-invariant with respect to $J$, i.e., $J\left(k e r F_{*}\right) \subseteq\left(k e r F_{*}\right)^{\perp}$. Then we say that $F$ is an anti-invariant Riemannian submersion.

On the other hand, in [34], we introduced semi-invariant submersions from almost Hermitian manifolds onto Riemannian manifolds as a generalization of holomorphic submersions and anti-invariant submersions, then we studied the geometry of

Received July 9, 2012, accepted September 25, 2012.

Communicated by Bang-Yen Chen.

2010 Mathematics Subject Classification: 53C15, 53C20, 53C43.

Key words and phrases: Submersion, Redundant robotic chain, Anti-invariant submersion, Slant submersion, Semi-invariant submersion. 
such maps. We recall that a Riemannian submersion $F$ from an almost Hermitian manifold $\left(M, J_{M}, g_{M}\right)$ with almost complex structure $J_{M}$ onto a Riemannian manifold $\left(N, g_{N}\right)$ is called a semi-invariant submersion if the fibers have differentiable distributions $D$ and $D^{\perp}$ such that $D$ is invariant with respect to $J_{M}$ and its orthogonal complementary distribution $D^{\perp}$ is totally real distribution, i.e, $J_{M}\left(D_{p}^{\perp}\right) \subseteq\left(k e r F_{* p}\right)^{\perp}$. Obviously, almost Hermitian submersions and anti-invariant submersions are semiinvariant submersions with $D^{\perp}=\{0\}$ and $D=\{0\}$, respectively.

Recently, we also introduced the notion of slant submersions from almost Hermitian manifolds onto arbitrary Riemannian manifolds[33] as follows: Let $F$ be a Riemannian submersion from an almost Hermitian manifold $\left(M_{1}, g_{1}, J_{1}\right)$ onto a Riemannian manifold $\left(M_{2}, g_{2}\right)$. If for any non-zero vector $X \in \Gamma\left(k e r F_{*}\right)$, the angle $\theta(X)$ between $J X$ and the space $k e r F_{*}$ is a constant, i.e. it is independent of the choice of the point $p \in M_{1}$ and choice of the tangent vector $X$ in $\operatorname{ker}_{*}$, then we say that $F$ is a slant submersion. In this case, the angle $\theta$ is called the slant angle of the slant submersion.

Moreover, Park and Prasad [29] defined semi-slant submersions from almost Hermitian manifolds as a generalization of slant submersions. They also investigated the geometry of such submersions and gave many nice examples.

We note that Riemannian submersions have been studied widely in different fields. In mathematical physics, in particular Kaluza-Klein theory, the general solution of a recent model can be expressed in terms of harmonic maps satisfying Einstein equations. However, a very general class of solutions is given by Riemannian submersions from the extra dimensional space onto the space in which the scalar fields take values. One can see details of this result in [15]. On the other hand, Einstein-Witten manifolds can be considered as the total space of the principal $\mathbf{S}^{1}$ - bundle over $\mathbf{C P}^{1} \times \mathbf{C P}^{1}$. Wang and Ziller [36] showed that the principal $\mathbf{S}^{1}$ - bundle over a product of Kähler-Einstein manifolds with positive first Chern class admits a totally geodesic Einstein metric that is unique up to scalling. The metric is determined by the property that the projection onto the base is a Riemannian submersion with totally geodesic fibers. Generalized EinsteinWitten manifolds are quotient manifolds of $\mathbf{S}^{3} \times \mathbf{S}^{5}$, where the $\mathbf{S}^{1}-$ action is given by $((z, w), y) \rightarrow\left(\left(e^{i l_{1} \theta} z, e^{i l_{2} \theta} w\right), e^{i l_{1} \theta} y\right)$, here $(z, w) \in \mathbf{S}^{3} \subseteq \mathbb{C}^{2}, y \in \mathbf{S}^{5}, l_{1}, l_{2}, k \in \mathbb{Z}$. We note that generalized Einstein-Witten manifolds are important in unified theory. Recently, Kennedy [21] investigated the existence of Einstein metrics on generalized Einstein-Witten manifolds by using Riemannian submersions. Another application for Riemannian submersions is statistical machine learning process. In [39], the authors used Riemannian submersions to employ a class specific information into a manifold. This can be viewed as a learning process in which the embedding is recovered from a labelled graph. Thus, as input to the training step, the method takes a set of graphs, whose nodes attributes are given by a set of vectors, and delivers, at output, a model which can be used for embedding graphs which are not in the training set. Riemannian submersions have been also used in medical imaging (as projections) [23], Statistical 
analysis on manifolds [4] and robotic theory[1] which will be explained in section 3 .

In this paper, we gather results on Riemannian submersions from almost Hermitian manifolds onto arbitrary Riemannian manifolds. Section 2 is devoted to the main results of geometry of Riemannian submersions. The results of this section can be found in [24]. Applications of Riemannian submersions are given in section 3. We note that applications of Riemannian submersions in mathematical physics were given in [6], [14] and [15] therefore here we give Altafini's results about applications of Riemannian submersions on redundant robotic chains as another application of such maps. In section 4, we define holomorphic submersions and recall Watson's result on such submersions. In section 5, we define invariant Riemannian submersions from almost Hermitian manifolds and show that although every holomorphic submersion is an invariant Riemannian submersion, the converse is not true. In section 6 , we define anti-invariant submersions from almost Hermitian manifolds, give an example, investigate the geometry of leaves of distributions and obtain the harmonicity of such submersions. We also show that anti-invariant Riemannian submersions are useful for obtaining decomposition theorems. In section 7, we introduce slant submersions, give examples and investigate the main properties of such submersions. In section 8 , we define semi-invariant submersions, give examples and examine the geometry of such submersions. Section 9 is devoted to the geometry of semi-slant submersions from almost Hermitian manifolds onto Riemannian manifolds. Finally, in section 10, we propose seven open problems to the readers.

\section{RIEMANNIAN SUBMERSIONS}

Riemannian submersions between Riemannian manifolds were studied by O'Neill [24] and [17]. Later such submersions were considered between manifolds endowed with (various) differentiable structures[15]. In this section we review basic information for Riemannian submersions from [24] and [15]. We first recall the definition of Riemannian submersions between Riemannian manifolds. Let $\left(M^{m}, g_{M}\right)$ and $\left(N^{n}, g_{N}\right)$ be Riemannian manifolds, where $\operatorname{dim}(M)=m$, $\operatorname{dim}(N)=n$ and $m>n$. A Riemannian submersion $F: M \longrightarrow N$ is a surjective map of $M$ onto $N$ satisfying the following axioms:

(S1) $F$ has maximal rank.

(S2) The differential $F_{*}$ preserves the lenghts of horizontal vectors.

For each $q \in N, F^{-1}(q)$ is an $(m-n)$ dimensional submanifold of $M$. The submanifolds $F^{-1}(q), q \in N$, are called fibers. A vector field on $M$ is called vertical if it is always tangent to fibers. A vector field on $M$ is called horizontal if it is always orthogonal to fibers. A vector field $X$ on $M$ is called basic if $X$ is horizontal and $F-$ related to a vector field $X_{*}$ on $N$, i.e., $F_{*} X_{p}=X_{* F(p)}$ for all $p \in M$. Note that we 
denote the projection morphisms on the distributions $\operatorname{ker} F_{*}$ and $\left(k e r f F_{*}\right)^{\perp}$ by $\mathcal{V}$ and $\mathcal{H}$, respectively.

We recall the following lemma from [24].

Lemma 2.1. Let $F: M \longrightarrow N$ be a Riemannian submersion between Riemannian manifolds and $X, Y$ be basic vector fields of $M$. Then

(a) $g_{M}(X, Y)=g_{N}\left(X_{*}, Y_{*}\right) \circ F$

(b) the horizontal part $[X, Y]^{\mathcal{H}}$ of $[X, Y]$ is a basic vector field and corresponds to $\left[X_{*}, Y_{*}\right]$, i.e., $F_{*}\left([X, Y]^{\mathcal{H}}\right)=\left[X_{*}, Y_{*}\right]$,

(c) $[V, X]$ is vertical for any vector field $V$ of $k e r F_{*}$,

(d) $\left(\nabla_{X}^{M} Y\right)^{\mathcal{H}}$ is the basic vector field corresponding to $\nabla_{X_{*}}^{N} Y_{*}$.

The geometry of Riemannian submersions is characterized by O'Neill's tensors $\mathcal{T}$ and $\mathcal{A}$ defined for vector fields $E, F$ on $M$ by

$$
\begin{aligned}
& \mathcal{A}_{E} F=\mathcal{H} \nabla_{\mathcal{H} E} \mathcal{V} F+\mathcal{V} \nabla_{\mathcal{H} E} \mathcal{H} F \\
& \mathcal{T}_{E} F=\mathcal{H} \nabla_{\mathcal{V} E} \mathcal{V} F+\mathcal{V} \nabla_{\mathcal{V} E} \mathcal{H} F
\end{aligned}
$$

where $\nabla$ is the Levi-Civita connection of $g_{M}$.

It is easy to see that a Riemannian submersion $F: M \longrightarrow N$ has totally geodesic fibers if and only if $\mathcal{T}$ vanishes identically. For any $E \in \Gamma(T M), \mathcal{T}_{E}$ and $\mathcal{A}_{E}$ are skew-symmetric operators on $(\Gamma(T M), g)$ reversing the horizontal and the vertical distributions. It is also easy to see that $\mathcal{T}$ is vertical, $\mathcal{T}_{E}=\mathcal{T}_{\mathcal{V} E}$ and $\mathcal{A}$ is horizontal, $\mathcal{A}=\mathcal{A}_{\mathcal{H} E}$. We note that the tensor fields $\mathcal{T}$ and $\mathcal{A}$ satisfy

$$
\begin{aligned}
& \mathcal{T}_{U} W=\mathcal{T}_{W} U, \forall U, W \in \Gamma\left(k e r F_{*}\right) \\
& \mathcal{A}_{X} Y=-\mathcal{A}_{Y} X=\frac{1}{2} \mathcal{V}[X, Y],
\end{aligned}
$$

for $X, Y \in \Gamma\left(\left(k e r F_{*}\right)^{\perp}\right)$.

On the other hand, from (1) and (2) we have

$$
\begin{aligned}
& \nabla_{V} W=\mathcal{T}_{V} W+\hat{\nabla}_{V} W \\
& \nabla_{V} X=\mathcal{H} \nabla_{V} X+\mathcal{T}_{V} X \\
& \nabla_{X} V=\mathcal{A}_{X} V+\mathcal{V} \nabla_{X} V \\
& \nabla_{X} Y=\mathcal{H} \nabla_{X} Y+\mathcal{A}_{X} Y
\end{aligned}
$$

for $X, Y \in \Gamma\left(\left(k e r F_{*}\right)^{\perp}\right)$ and $V, W \in \Gamma\left(k e r F_{*}\right)$, where $\hat{\nabla}_{V} W=\mathcal{V} \nabla_{V} W$. If $X$ is basic, then $\mathcal{H} \nabla_{V} X=\mathcal{A}_{X} V$. 
We now state the following curvature relation between the base manifold and the total manifold [24].

Theorem 2.1. For $X, Y \in \Gamma\left(\left(k e r F_{*}\right)^{\perp}\right)$, we have

$$
\begin{aligned}
g_{N}\left(R^{N}\left(X^{\prime}, Y^{\prime}\right) X^{\prime}, Y^{\prime}\right)= & g_{M}\left(R^{M}(X, Y) X, Y\right) \\
& +\frac{3}{4} g_{M}\left([X, Y]^{\mathcal{V}},[X, Y]^{\mathcal{V}}\right),
\end{aligned}
$$

where $X^{\prime}=F_{*} X, Y^{\prime}=F_{*} Y$. In other words, the sectional curvature in the base manifold increases by the amount $\frac{3}{4} g_{M}\left([X, Y]^{\mathcal{V}},[X, Y]^{\mathcal{V}}\right)$.

We now recall the notion of harmonic maps between Riemannian manifolds. Let $\left(M, g_{M}\right)$ and $\left(N, g_{N}\right)$ be Riemannian manifolds and suppose that $\varphi: M \longrightarrow N$ is a smooth mapping between them. Then the differential $\varphi_{*}$ of $\varphi$ can be viewed a section of the bundle $\operatorname{Hom}\left(T M, \varphi^{-1} T N\right) \longrightarrow M$, where $\varphi^{-1} T N$ is the pullback bundle which has fibers

$$
\left(\varphi^{-1} T N\right)_{p}=T_{\varphi(p)} N,
$$

$p \in M$. $\operatorname{Hom}\left(T M, \varphi^{-1} T N\right)$ has a connection $\nabla$ induced from the Levi-Civita connection $\nabla^{M}$ and the pullback connection. Then the second fundamental form of $\varphi$ is given by

$$
\nabla \varphi_{*}(X, Y)=\nabla_{X}^{\varphi} \varphi_{*}(Y)-\varphi_{*}\left(\nabla_{X}^{M} Y\right)
$$

for $X, Y \in \Gamma(T M)$, where $\nabla^{\varphi}$ is the pullback connection. It is known that the second fundamental form is symmetric. For a Riemannian submersion $F$, one can easily obtain

$$
\left(\nabla F_{*}\right)(X, Y)=0
$$

for $X, Y \in \Gamma\left(\left(k e r F_{*}\right)^{\perp}\right)$. A smooth map $\varphi:\left(M, g_{M}\right) \longrightarrow\left(N, g_{N}\right)$ is said to be harmonic if $\operatorname{trace} \nabla \varphi_{*}=0$. On the other hand, the tension field of $\varphi$ is the section $\tau(\varphi)$ of $\Gamma\left(\varphi^{-1} T N\right)$ defined by

$$
\tau(\varphi)=\operatorname{div} \varphi_{*}=\sum_{i=1}^{m} \nabla \varphi_{*}\left(e_{i}, e_{i}\right),
$$

where $\left\{e_{1}, \ldots, e_{m}\right\}$ is the orthonormal frame on $M$. Then it follows that $\varphi$ is harmonic if and only if $\tau(\varphi)=0$, for details, see [2].

\section{Applications of Riemannian Submersions}

Riemannian submersions have their applications in Kaluza-Klein theory [15], [6], statistical machine learning process [39] medical imaging [23], statistical analysis on 
manifolds [4] and the theory of robotics [1]. In Kaluza-Klein theory, the general solution of a recent model can be expressed in terms of harmonic maps satisfying Einstein equations. However, a very general class of solutions is given by Riemannian submersions from the extra dimensional space onto the space in which the scalar fields take values. Applications of Riemannian submersions in Kaluza-Klein theory have been given in details in the book [15] and papers [14] and [6]. Therefore, we do not repeat applications of Riemannian submersions in Kaluza-Klein theory here. Of course it is out of scope of this paper to mention all applications of Riemannian submersions, but we will focus on applications of Riemannian submersions in redundant robotic chains given in [1].

\subsection{Preliminaries}

A particle is an object with mass concentrated at a point. A rigid body is an object with mass and volume. A free mechanical system is a collection $P_{1}, \ldots, P_{N}$ of particles and $B_{1}, \ldots, B_{N}$ of rigid bodies which move independently of one another. To specify the location of a particle, choose an inertial reference frame $\left(O_{\text {spatial }},\left\{s_{1}, s_{2}, s_{3}\right\}\right)$ consisting of a spatial origin $O_{\text {spatial }}$ and an orthonormal frame $\left\{s_{1}, s_{2}, s_{3}\right\}$ at $O_{\text {spatial }}$. The position of the particle $P_{j}$ is exactly determined by a vector $r_{j} \in R^{3}$ from $O_{\text {spatial }}$ to the location of $P_{j}$. The configuration of a free mechanical system is specified by a point in

$$
\underbrace{R^{3} \times \ldots R^{3}}_{N_{P} \text { copies }} \times \underbrace{\left(S O(3) \times R^{3}\right) \times \ldots\left(S O(3) \times R^{3}\right)}_{N_{B} \text { copies }} .
$$

Robot manipulators can be regarded as open-loop link mechanisms consisting of several links connected together by joints. Joints are typically rotary or linear. A rotary joint is like a hinge and allows relative rotation between two links. A linear joint allows a linear relative motion between two links. Each joint represents the interconnection between two links. A configuration of a manipulator is a complete specification of the location of every point on the manipulator. The set of all possible configurations is called the configuration space. If we know the values for the joint variables (i.e., the joint angle for revolute joints, or the joint offset for prismatic joints), then it is straightforward to infer the position of any point on the manipulator, since the individual links of the manipulator are assumed to be rigid, and the base of the manipulator is assumed to be fixed.

As an example, for two link manipulator, a planar joint can be built from a revolute joint attached to two independent prismatic joints. The motion of a planar joint is restricted to $S E(2)$, regarded as a $3-$ dimensional subgroup of $S E(3)$.

Here we have

$$
\left(S O(3) \times R^{3}\right) \times\left(S O(3) \times R^{3}\right) .
$$

However, the actual configurations of the system are specified by the angles $\theta_{1}$ and $\theta_{2}$ 
as shown in the figure. Each angle is measured by a point on the circle

$$
S^{1}=\left\{(x, y) \in R^{2} \mid x^{2}+y^{2}=1\right\} .
$$

Thus the configurations of this simple two link robot are specified by a point in $S^{1} \times S^{1}$.

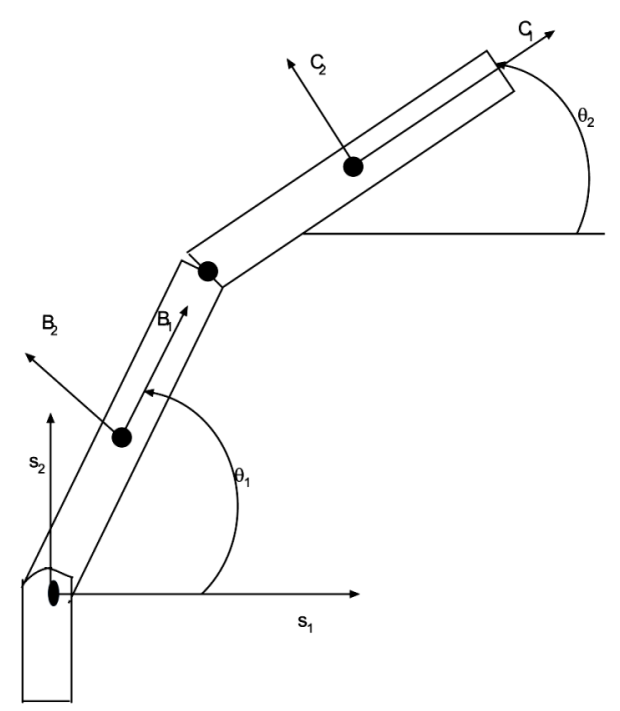

Fig. 1. Two-link planar manipulator:

An object is said to have $\mathrm{n}$ degrees-of-freedom (DOF) if its configuration can be minimally specified by $n$ parameters. Thus, the number of DOF is equal to the dimension of the configuration space. For a robot manipulator, the number of joints determines the number DOF. A rigid object in three-dimensional space has six DOF: three for positioning and three for orientation (e.g., roll, pitch and yaw angles). Therefore, a manipulator should typically possess at least six independent DOF. With fewer than six DOF the arm cannot reach every point in its work environment with arbitrary orientation. Certain applications such as reaching around or behind obstacles may require more than six DOF. A manipulator having more than six links is referred to as a kinematically redundant manipulator. The workspace of a manipulator is the total volume swept out by the endeffector as the manipulator executes all possible motions. The workspace is constrained by the geometry of the manipulator as well as mechanical constraints on the joints. The workspace is often broken down into a reachable workspace and a dexterous workspace. The reachable workspace is the entire set of points reachable by the manipulator, whereas the dexterous workspace consists of those points that the manipulator can reach with an arbitrary orientation of the end-effector. Obviously the dexterous workspace is a subset of the reachable workspace, for details see: [35] and [7]. 


\subsection{Forward kinematics map as an example of Riemannian submersions}

This subsection is taken from a paper by Altafini [1] which may be consulted for the details we can not discuss in this paper. The forward kinematics of a robot arm is represented by the smooth map

$$
\begin{aligned}
\rho: Q & \rightarrow S E(3) \\
q=\left[q_{1}, \ldots, q_{n}\right] & \rightarrow g=\rho(q),
\end{aligned}
$$

where $S E(3)$ is the Lie group of rigid body motions in 3- dimensional space. Each joint variable leaves on $\mathbb{S}$ or on $\mathbb{R}$ and therefore lies in the left/right invariant translation of a one dimensional subgroup of $S E(3)$. Hence their product $Q$ is an $n$-dimensional abelian group. The movements of the end-effector are the resulting of the composition of rototranslatins of the $n-$ one parameter joints. This can be represented as a product of exponentials of the single one-degree of freedom screw motion. The interpration of that method is the following. One fixes a coordinate system on $Q$ and one on $S E(3)$ and identifies them through $\rho$. Now differentiating (11), we have

$$
\begin{aligned}
\rho_{*}^{B}: T Q & \rightarrow T S E(3) \\
(q, \dot{q}) & \rightarrow\left(g, g X_{\rho(g)}\right)=\left(g, g J^{b}(q) \dot{q}\right),
\end{aligned}
$$

where $\dot{q}$ corresponds to the velocity in coordinates $\dot{q}=\dot{q} \frac{\partial}{\partial q}$.

Using the natural paralelism of an abelian group, instead of tangent bundle $\rho_{*}^{B}$ one can consider the map between tangent space $\rho_{*}$, which is called the differential forward kinematics

$$
\begin{aligned}
\rho_{*}: T_{q} Q & \rightarrow \mathfrak{s e}(3) \\
\dot{q} & \rightarrow X_{\rho(g)}=J^{b}(q) \dot{q},
\end{aligned}
$$

where $J^{b}$ is the Jacobian of the product of exponentials.

In joint space $Q$, it is possible to construct a positive definite and symmetric metric $M(q)$ by

$$
M(q)=\sum_{i=1}^{9} J_{i}^{T}(q) M_{i} J_{i}(q),
$$

where $M_{i}$ is the quadratic form representing the generalized inertia tensor of the $i-$ th joint and $J_{i}(q)$ is the $\mathbb{R}^{6}$ - velocity of the $j$ - th joint $(j \leq i)$ which is referred to the $i$ - th link. Consequently $Q$ is a Riemannian manifold.

Moreover the Levi-Civita connection determined by $M$ is locally flat. For flat manifolds, it is possible to construct an isometry

$$
\varphi:(Q, M(q)) \longrightarrow(\tilde{Q}, I)
$$


such that

$$
\begin{aligned}
<\dot{\tilde{q}}, \dot{\tilde{q}}>_{\tilde{Q}} & =\varphi_{*}^{T}(q) \varphi_{*}(q) \\
& =\dot{q}^{T} N^{T}(q) N(q) \dot{q} \\
& =<\dot{q}, \dot{q}>_{Q},
\end{aligned}
$$

where $N(q)$ is the Jacobian of the isometry. Then $M(q)$ can be written as

$$
M(q)=N(q)^{T} N(q) .
$$

Thus the metric of $Q$ can be reduced to the identity.

Now assume the following condition:

R1 Assume that at $q \in Q, \operatorname{rank}\left(\rho_{*}(q)\right)=6$.

Since the dimension of $S E(3)$ is 6 , this assumption implies that we have a 6 degree of freedom manipulator.

It is known that in $S E(3)$, there is no ad-invariant Riemannian metric which implies that there is no natural way of transporting vector fields between points of $S E(3)$ and that there is no natural concept of distance on $S E(3)$. The two most common approaches to tackle this obstruction are: (1) Ad-invariant pseudo Riemannian structure, (2) Double geodesic. The double geodesic method is based on discarding the group structure of $S E(3)$ and consider separately the bi-invariant metric of $S O(3)$ and the Euclidean metric of $\mathbb{R}^{3}$. The corresponding quadratic form is

$$
M_{d g}=\left(\begin{array}{cc}
I & 0 \\
0 & I
\end{array}\right) .
$$

In fact, above quadratic form is special. One can choose a more general form, but this will be enough for our aim. The Riemannian connection is defined by

$$
\tilde{\nabla}_{g A_{i}}\left(g A_{j}\right)=g \tilde{\nabla}_{A_{i}} A_{j}=\Gamma_{i j}^{k} g A_{k}
$$

for all $g \in S E(3)$ and $A_{i}$ the elements of an orthonormal basis of left invariant vector fields. Since $Q$ is a Riemannian manifold, at each $q \in Q$, we have the following decomposition

$$
T_{q} Q=\mathcal{H}_{q} \oplus \mathcal{V}_{q},
$$

where $\mathcal{V}_{q}$ is the kernel space of $\rho_{*}(q)$ and $\mathcal{H}_{q}$ is the orthogonal complementary subspace to $\mathcal{V}_{q}$ in $T_{q} Q$.

$\mathbf{R 2}$ Assume that at $q \in Q, \operatorname{dim} \mathcal{H}_{q}=6$ and $\operatorname{dim}_{q}=n-6$.

Now we have the following result. 
Proposition 3.1. [1]. Under the assumption R2, the forward kinematic map is a Riemannian submersion.

Proof. For the orthogonal subalgebra of $\mathfrak{s e}(3)=\mathfrak{s o}(3)\left(\mathbb{S}^{3}, \mathfrak{s o}(3)\right.$ is isomorphic to $\left(\mathbb{R}^{3}, \times\right)$. The cross product induces a Lie algebra structure on $\mathbb{R}^{3}$ which is compatible with the Euclidean inner product, i.e., for $x, y \in \mathbb{R}^{3},<x, y>_{\mathbb{R}^{3}}=<\tilde{x}, \tilde{y}>_{\mathfrak{s o}(3)}$. Now for $\dot{q}_{X}, \dot{q}_{Y} \in \mathcal{H}_{q}$, from (16) we have

$$
<\dot{q}_{X}, \dot{q}_{Y}>_{\mathcal{Q}}=\dot{q}_{X}^{T} N^{T}(q) N(q) \dot{q}_{Y} .
$$

Using (17) and (14) we get

$$
<\dot{q}_{X}, \dot{q}_{Y}>_{\mathcal{Q}}=\dot{q}_{X}^{T} J_{i}^{T}\left(q_{X}\right) M_{i} J_{i}\left(q_{Y}\right) \dot{q}_{Y} .
$$

On the other hand, from (13) and (18) we have

$$
<\dot{q}_{X}, \dot{q}_{Y}>_{\mathfrak{s e}(3)}=\dot{q}_{X}^{T} J^{b}\left(q_{X}\right) M_{d g} J_{b}\left(q_{Y}\right) \dot{q}_{Y} .
$$

Then since the metric of $Q$ is reduced to the identity by the isometry $\varphi$, proof is complete due to (20) and (21).

In this construction, since $Q$ is Abelian, the horizontal distribution is also integrable. Also note that, in robotics, $\rho^{-1}(g)$ is the set of joint movements that do not affect the end effector.

\section{HOLOMORPHIC SUBMERSIONS}

In this section, we recall main results for holomorphic or almost Hermitian submersions which were defined by Watson in [37]. Let $(\bar{M}, g)$ be an almost Hermitian manifold. This means [38] that $\bar{M}$ admits a tensor field $J$ of type $(1,1)$ on $\bar{M}$ such that, $\forall X, Y \in \Gamma(T \bar{M})$, we have

$$
J^{2}=-I, \quad g(X, Y)=g(J X, J Y) .
$$

An almost Hermitian manifold $\bar{M}$ is called Kähler manifold if

$$
\left(\bar{\nabla}_{X} J\right) Y=0, \forall X, Y \in \Gamma(T \bar{M}),
$$

where $\bar{\nabla}$ is the Levi-Civita connection on $\bar{M}$.

A smooth map $\phi: M \longrightarrow N$ between almost complex manifolds $(M, J)$ and $(N, \bar{J})$ is called almost complex (or holomorphic) map if $\phi_{*}(J X)=\bar{J} \phi_{*}(X)$ for $X \in \Gamma(T M)$, where $J$ and $\bar{J}$ are complex structures of $M$ and $N$, respectively.

Let $M$ be a complex $m$ - dimensional almost Hermitian manifold with Hermitian metric $g_{M}$ and almost complex structure $J_{M}$ and $N$ be a complex $n$ - dimensional almost Hermitian manifold with almost complex structure $J_{N}$, Watson [37] considered 
the holomorphic Riemannian submersion, called Hermitian submersion, and obtained the fundamental properties of this map. Later, this topic was studied by many authors. More precisely, a Riemannian submersion $F: M \longrightarrow N$ is called an almost Hermitian submersion if $F$ is an almost complex mapping. The main result of this notion is that the vertical and horizontal distributions are $J_{M}-$ invariant. In most cases, the base manifold and each fiber have the same kind of structure as the total space. Watson also found the following result.

Theorem 4.1. [37]. Let $F:\left(M, g_{M}, J_{M}\right) \longrightarrow\left(N, g_{N}, J_{N}\right)$ be a holomorphic submersion from an almost Hermitian manifold $\left(M, g_{M}, J_{M}\right)$ onto an almost Hermitian manifold $\left(N, g_{N}, J_{N}\right)$. Then

(a) If the total space is almost semi-Kähler, then the base manifold is almost semiKähler if and only if each fiber is minimal.

(b) A quasi-Kähler submersion is curvature decreasing.

(c) An almost semi-Kähler submersion is Betti number decreasing.

We note that the idea of holomorphic submersions between almost Hermitian manifolds has been extended to almost contact manifolds [12], locally conformal Kähler manifolds [22], quaternion Kähler manifolds [20] and other manifolds equipped with a structure, see [15].

\section{InVARIant RiemanNian Submersions}

In previous section, we have seen that the notion of the holomorphic submersions implies that the vertical distributions and horizontal distributions are invariant under the complex structure. In this section, we consider the converse. More precisely, if the vertical and horizontal distributions of a submersion between almost Hermitian manifolds are invariant with respect to the complex structure of the total manifold, then is this Riemannian submersion a holomorphic map? In fact, we are going to to show that an invariant submersion may not be a holomorphic submersion. We first present the following definition.

Definition 5.1. Let $F$ be a Riemannian submersion from an almost Hermitian manifold $\left(M, g_{M}, J_{M}\right)$ onto a Riemannian manifold $\left(N, g_{N}, J_{N}\right)$. Then we say that $F$ is an invariant submersion if the vertical distribution is invariant with respect to $J_{M}$, i.e.,

$$
J_{M}\left(k e r F_{*}\right)=\operatorname{ker} F_{*} .
$$

From above definition, we have the following result.

Corollary 5.1. Let $F$ be a Riemannian submersion from an almost Hermitian manifold $\left(M, g_{M}, J_{M}\right)$ onto a Riemannian manifold $\left(N, g_{N}, J_{N}\right)$. Then the horizontal distribution is invariant with respect to $J_{M}$. 
Here is an example of an invariant Riemannian submersion, it also shows that an invariant submersion between almost Hermitian manifolds is not a holomorphic map.

Example 5.1. Let $F: \mathbb{R}^{8} \longrightarrow \mathbb{R}^{4}$ be a map defined by

$$
F\left(x_{1}, x_{2}, x_{3}, x_{4}, x_{5}, x_{6}, x_{7}, x_{8}\right)=\left(\frac{x_{1}-x_{5}}{\sqrt{2}}, \frac{x_{2}-x_{6}}{\sqrt{2}}, \frac{x_{3}-x_{7}}{\sqrt{2}}, \frac{x_{4}-x_{8}}{\sqrt{2}}\right) .
$$

Then, by direct calculations

$$
k e r F_{*}=\operatorname{span}\left\{Z_{1}, Z_{2}, Z_{3}, Z_{4}\right\}
$$

where

$$
\begin{array}{ll}
Z_{1}=\partial x_{1}+\partial x_{5} & , \quad Z_{2}=\partial x_{2}+\partial x_{6} \\
Z_{3}=\partial x_{3}+\partial x_{7} & , \quad Z_{4}=\partial x_{4}+\partial x_{8}
\end{array}
$$

and

$$
\left(k e r F_{*}\right)^{\perp}=\operatorname{span}\left\{H_{1}, H_{2}, H_{3}, H_{4}\right\}
$$

where

$$
\begin{array}{ll}
H_{1}=\partial x_{1}-\partial x_{5} \quad & H_{2}=\partial x_{2}-\partial x_{6} \\
H_{3}=\partial x_{3}-\partial x_{7} & , \quad H_{4}=\partial x_{4}-\partial x_{8} .
\end{array}
$$

Then considering complex structures on $\mathbb{R}^{8}$ and $\mathbb{R}^{4}$ defined by

$$
J^{8}\left(a_{1}, a_{2}, a_{3}, a_{4}, a_{5}, a_{6}, a_{7}, a_{8}\right)=\left(-a_{2}, a_{1},-a_{4}, a_{3},-a_{6}, a_{5},-a_{8}, a_{7}\right)
$$

and

$$
J^{4}\left(a_{1}, a_{2}, a_{3}, a_{4}\right)=\left(-a_{3},-a_{4}, a_{1}, a_{2}\right),
$$

it is easy to see that $k e r F_{*}$ and $\left(k e r F_{*}\right)^{\perp}$ are invariant with respect to $J^{8}$. Thus $F$ is an invariant submersion. However, $F$ is not a holomorphic submersion. Indeed, for $H_{1}$, we have $J^{4} F_{*}\left(H_{1}\right)=\sqrt{2} \partial y_{3}$ and $F_{*} J^{8}\left(H_{1}\right)=\sqrt{2} \partial y_{2}$, i.e., $J^{4} F_{*}\left(H_{1}\right) \neq F_{*}\left(J^{8} H_{1}\right)$.

Thus we can state the following.

Proposition 5.1. Every holomorphic submersion is an invariant submersion. However, an invariant submersion may not be a holomorphic submersion.

For an invariant Riemannian submersion we have the following proposition:

Proposition 5.2. Let $F$ be an invariant submersion from a Kähler manifold $\left(M, g_{M}\right.$, $\left.J_{M}\right)$ onto a Riemannian manifold $\left(N, g_{N}\right)$. Then the fibers are minimal submanifolds. 
Proof. Since $k e r F_{*}$ is invariant with respect to $J_{M}$, the fibers are minimal if and only if $\mathcal{T}_{U} U+\mathcal{T}_{J_{M} U} J_{M} U=0$ for $U \in \operatorname{ker} F_{*}$. Using (5) and Kähler character of $M$ we have

$$
g_{M}\left(\mathcal{T}_{U} U+\mathcal{T}_{J_{M} U} J_{M} U, X\right)=g_{M}\left(\nabla_{U} U, X\right)-g_{M}\left(\nabla_{J_{M} U} U, J_{M} X\right)
$$

for $X \in \Gamma\left(\left(k e r F_{*}\right)^{\perp}\right)$. Then, Lemma 1.1 (c) and (22) imply

$$
g_{M}\left(\mathcal{T}_{U} U+\mathcal{T}_{J_{M} U} J_{M} U, X\right)=0
$$

which shows that $\mathcal{T}_{U} U+\mathcal{T}_{J_{M} U} J_{M} U=0$ due to $\mathcal{T}$ reverses horizontal and vertical distributions.

\section{Anti-invariant Riemannian Submersions from Almost Hermitian Manifolds}

In this section, we define anti-invariant Riemannian submersions from almost Hermitian manifolds onto Riemannian manifolds. For details see [32].

Definition 6.1. [32]. Let $M$ be a complex $m$ - dimensional almost Hermitian manifold with Hermitian metric $g_{M}$ and almost complex structure $J$ and $N$ be a Riemannian manifold with Riemannian metric $g_{N}$. Suppose that there exists a Riemannian submersion $F: M \longrightarrow N$ such that $\operatorname{ker}_{*}$ is anti-invariant with respect to $J$, i.e., $J\left(k e r F_{*}\right) \subseteq\left(k e r F_{*}\right)^{\perp}$. Then we say that $F$ is an anti-invariant Riemannian submersion.

First of all, from above definition, we have $J\left(k e r F_{*}\right)^{\perp} \cap k e r F_{*} \neq\{0\}$. We denote the complementary orthogonal subbundle to $J\left(k e r F_{*}\right)$ in $\left(k e r F_{*}\right)^{\perp}$ by $\mu$. Then we have

$$
\left(k e r F_{*}\right)^{\perp}=J k e r F_{*} \oplus \mu .
$$

It is easy to see that $\mu$ is an invariant subbundle of $\left(k e r F_{*}\right)^{\perp}$, under the endomorphism $J$. Thus, for $X \in \Gamma\left(\left(k e r F_{*}\right)^{\perp}\right)$, we have

$$
J X=B X+C X,
$$

where $B X \in \Gamma\left(k e r F_{*}\right)$ and $C X \in \Gamma(\mu)$.

Example 6.1. Let $F: R^{4} \longrightarrow R^{2}$ be a map defined by $F\left(x_{1}, x_{2}, x_{3}, x_{4}\right)=$ $\left(\frac{x_{1}+x_{4}}{\sqrt{2}}, \frac{x_{2}+x_{3}}{\sqrt{2}}\right)$. Then, by direct calculations

$$
\operatorname{ker} F_{*}=\operatorname{span}\left\{Z_{1}=\partial x_{1}-\partial x_{4}, Z_{2}=\partial x_{2}-\partial x_{3}\right\}
$$

and

$$
\left(k e r F_{*}\right)^{\perp}=\operatorname{span}\left\{X_{1}=\partial x_{1}+\partial x_{4}, X_{2}=\partial x_{2}+\partial x_{3}\right\} .
$$


Then it is easy to see that $F$ is a Riemannian submersion. Moreover, $J Z_{1}=X_{2}$ and $J Z_{2}=X_{1}$ imply that $J\left(k e r F_{*}\right)=\left(k e r F_{*}\right)^{\perp}$. As a result, $F$ is an anti-invariant Riemannian submersion.

In the sequel, we find necessary and sufficient conditions in terms of (24), (25) and the second fundamental form (9) for the distribution $\left(k e r F_{*}\right)^{\perp}$ to be integrable. Note that the distribution $k e r F_{*}$ is always integrable.

Theorem 6.1. [32]. Let $F$ be an anti-invariant Riemannian submersion from a Kähler manifold $\left(M, g_{M}, J\right)$ onto a Riemannian manifold $\left(N, g_{N}\right)$. Then the following assertions are equivalent to each other;

(a) $\left(k e r F_{*}\right)^{\perp}$ is integrable.

(b)

$$
\begin{aligned}
g_{N}\left(\left(\nabla F_{*}\right)(Y, B X), F_{*} J V\right)= & g_{M}\left(C Y, J \mathcal{A}_{X} V\right) \\
& -g_{M}\left(C X, J \mathcal{A}_{Y} V\right) \\
& +g_{N}\left(\left(\nabla F_{*}\right)(X, B Y), F_{*} J V\right) .
\end{aligned}
$$

(c) $g_{M}\left(\mathcal{A}_{Y} B X-\mathcal{A}_{X} B Y, J V\right)=g_{M}\left(C Y, J \mathcal{A}_{X} V\right)-g_{M}\left(C X, J \mathcal{A}_{Y} V\right)$, for $X, Y \in \Gamma\left(\left(k e r F_{*}\right)^{\perp}\right)$ and $V \in \Gamma\left(k e r F_{*}\right)$.

We say that an anti-invariant Riemannian submersion is a Lagrangian Riemannian submersion if $J\left(k e r F_{*}\right)=\left(k e r F_{*}\right)^{\perp}$. From above theorem, we have the following corollary.

Corollary 6.1. [32]. Let $F:\left(M, g_{M}, J\right) \longrightarrow\left(N, g_{N}\right)$ be a Lagrangian Riemannian submersion. Then the following assertions are equivalent to each other;

(a) $\left(\text { ker } F_{*}\right)^{\perp}$ is integrable.

(b) $\left.\left(\nabla F_{*}\right)(X, J Y)=\left(\nabla F_{*}\right)(J X, Y)\right)$ for $X, Y \in \Gamma\left(\left(k e r F_{*}\right)^{\perp}\right)$.

(c) $\mathcal{A}_{X} J Y=\mathcal{A}_{Y} J X$.

The following theorems show that anti-invariant Riemannian submersions produce new conditions for the geometry of leaves of the horizontal and vertical distributions.

Theorem 6.2. [32]. Let $F$ be an anti-invariant Riemannian submersion from a Kähler manifold $\left(M, g_{M}, J\right)$ onto a Riemannian manifold $\left(N, g_{N}\right)$. Then the following assertions are equivalent to each other;

(a) $\left(k e r F_{*}\right)^{\perp}$ defines a totally geodesic foliation on $M$.

(b) $g_{M}\left(\mathcal{A}_{X} B Y, J V\right)=g_{M}\left(C Y, J \mathcal{A}_{X} V\right)$.

(c) $g_{N}\left(\left(\nabla F_{*}\right)(X, J Y), F_{*} J V\right)=g_{M}\left(C Y, J \mathcal{A}_{X} V\right)$. for $X, Y \in \Gamma\left(\left(k e r F_{*}\right)^{\perp}\right)$. 
For the distribution $\operatorname{ker} F_{*}$, we have;

Theorem 6.3. [32]. Let $F:\left(M, g_{M}, J\right) \longrightarrow\left(N, g_{N}\right)$ be a Lagrangian Riemannian submersion, where $\left(M, g_{M}\right)$ is Kähler manifold and $\left(N, g_{N}\right)$ is a Riemannain manifold. Then the following assertions are equivalent to each other;

(a) $\left(k e r F_{*}\right)$ defines a totally geodesic foliation on $M$.

(b) $g_{N}\left(\left(\nabla F_{*}\right)(Z, J X), F_{*} J V\right)=0$ for $X \in \Gamma\left(\left(k e r F_{*}\right)^{\perp}\right)$ and $Z, W \in \Gamma\left(k e r F_{*}\right)$.

(c) $\mathcal{T}_{Z} J W=0$.

We also have the following corollary.

Corollary 6.2. [32]. Let $F$ be a Lagrangian Riemannian submersion from a Kähler manifold $\left(M, g_{M}, J\right)$ to a Riemannian manifold $\left(N, g_{N}\right)$. Then the following assertions are equivalent to each other;

(a) $\left(\text { ker } F_{*}\right)^{\perp}$ defines a totally geodesic foliation on $M$.

(b) $\mathcal{A}_{X} J Y=0$.

(c) $\left(\nabla F_{*}\right)(X, J Y)=0$ for $X, Y \in \Gamma\left(\left(k e r F_{*}\right)^{\perp}\right)$.

It is known that the tensor $\mathcal{T}$ behaves as the second fundamental form of fibers of a Riemannian submersion. Thus fibers are totally geodesic if and only if $\mathcal{T}_{U} V=0$ for $U, V \in \Gamma\left(\operatorname{ker} F_{*}\right)$. Hence, it follows that a Riemannian submersion is totally geodesic if and only if $\mathcal{T}_{U} V=0$ and the horizontal distribution is parallel due to $\left(\nabla F_{*}\right)(X, Y)=0$ for $X, Y \in \Gamma\left(\left(k e r F_{*}\right)^{\perp}\right)$. Next we show that the notion of anti-invariant submersions present new conditions for a Riemannian submersion to be totally geodesic.

Theorem 6.4. [32]. Let $F$ be a Lagrangian Riemannian submersion from a Kähler manifold $\left(M, g_{M}, J\right)$ to a Riemannian manifold $\left(N, g_{N}\right)$. Then $F$ is a totally geodesic map if and only if

$$
\mathcal{T}_{W} J V=0, \forall W, V \in \Gamma\left(k e r F_{*}\right)
$$

and

$$
\mathcal{A}_{X} J W=0, \forall X \in \Gamma\left(\left(k e r F_{*}\right)^{\perp}\right) .
$$

Moreover, we also have a new result to characterize the harmonicity of Riemannian submersions by using the notion of Lagrangian submersion.

Theorem 6.5. [32]. Let $F$ be a Lagrangian Riemannian submersion from a Kähler manifold $\left(M, g_{M}, J\right)$ to a Riemannian manifold $\left(N, g_{N}\right)$. Then $F$ is harmonic if and only if Trace $J \mathcal{T}_{V}=0$ for $V \in \Gamma\left(k e r F_{*}\right)$. 
Let $g$ be a Riemannian metric tensor on the manifold $M=B \times F$ and assume that the canonical foliations $D$ and $\bar{D}$ intersect perpendicularly everywhere. Then from de Rham's theorem [13], we know that $g$ is the metric tensor of a usual product Riemannian manifold if and only if $D$ and $\bar{D}$ are totally geodesic foliations. This notion later has been extended to the other product manifolds. The concept of warped products was first introduced by Bishop and O'Neill [5] to construct examples of Riemannian manifolds with negative curvature. Many spacetime models are examples of warped product manifolds. More precisely, Robertson-Walker spacetimes, Asymptotically flat spacetimes, Schwarzschild spacetimes and Reissner-Nordström spacetimes are examples of warped product manifolds, for details, [18].

Let $\left(B, g_{1}\right)$ and $\left(F, g_{2}\right)$ be two Riemannian manifolds, $f: B \rightarrow(0, \infty)$ and $\pi: B \times F \rightarrow B, \eta: B \times F \rightarrow F$ the projection maps given by $\pi(p, q)=p$ and $\eta(p, q)=q$ for every $(p, q) \in B \times F$. The warped product $M=B \times F$ is the manifold $B \times F$ equipped with the Riemannian structure such that

$$
g(X, Y)=g_{1}\left(\pi_{*} X, \pi_{*} Y\right)+(f \circ \pi)^{2} g_{2}\left(\eta_{*} X, \eta_{*} Y\right)
$$

for every $X$ and $Y$ of $M$ and $*$ is symbol for the tangent map. The function $f$ is called the warping function of the warped product manifold. In particular, if the warping function is constant, then the manifold $M$ is said to be trivial. Let $\left(B, g_{B}\right)$ and $\left(F, g_{F}\right)$ be Riemannian manifolds of dimensions $m$ and $n$, respectively and let $\pi: B \times F \rightarrow B$ and $\sigma: B \times F \rightarrow F$ be the canonical projections. Also let $f: B \times F \rightarrow(0, \infty)$ be a smooth function. Then the twisted product [30]) of $\left(B, g_{B}\right)$ and $\left(F, g_{F}\right)$ with twisting functions $b$ and $f$ is defined to be the product manifold $M=B \times F$ with metric tensor $g=g_{B} \oplus f^{2} g_{F}$ given by $g=f^{2} g_{B}+b^{2} g_{F}$. Next theorem shows that the existence of anti-invariant submersions implies decomposition structure on the total manifold. More precisely we have the following results.

Theorem 6.6. [32]. Let $F$ be a Lagrangian Riemannian submersion from a Kähler manifold $\left(M, g_{M}, J\right)$ to a Riemannian manifold $\left(N, g_{N}\right)$. Then $M$ is a locally product manifold if and only if $\mathcal{A}_{X} J Y=0$ and $\mathcal{T}_{V} J W=0$ for $X, Y \in \Gamma\left(\left(k e r F_{*}\right)^{\perp}\right)$ and $V, W \in \Gamma\left(k e r F_{*}\right)$.

Theorem 6.7. [32]. Let $F$ be a Lagrangian Riemannian submersion from a Kähler manifold $\left(M, g_{M}, J\right)$ to a Riemannian manifold $\left(N, g_{N}\right)$. Then $M$ is a locally twisted product manifold of the form $M_{\left(k e r F_{*}\right)} \times_{f} M_{k e r F_{*}}$ if and only if

$$
\mathcal{T}_{V} J X=-g_{M}\left(X, \mathcal{T}_{V} V\right)\|V\|^{-2} J V
$$

and

$$
\mathcal{A}_{X} J Y=0
$$

for $X \in \Gamma\left(\left(k e r F_{*}\right)^{\perp}\right)$ and $V \in \Gamma\left(k e r F_{*}\right)$, where $M_{\left(k e r F_{*}\right)^{\perp}}$ and $M_{k e r F_{*}}$ are integral manifolds of the distributions $\left(k e r F_{*}\right)^{\perp}$ and $k e r F_{*}$. 


\section{Slant Submersions from Almost Hermitian Manifolds}

In this section, we define slant submersions from almost Hermitian manifolds onto Riemannian manifolds by using the definition of a slant distribution given in [8]. Then we investigate the harmonicity of a slant submersions and obtain a decomposition theorem for the total manifold.

Definition 7.1. [33]. Let $F$ be a Riemannian submersion from an almost Hermitian manifold $\left(M_{1}, g_{1}, J_{1}\right)$ onto a Riemannian manifold $\left(M_{2}, g_{2}\right)$. If for any non-zero vector $X \in \Gamma\left(k e r F_{*}\right)$, the angle $\theta(X)$ between $J_{1} X$ and the space $k e r F_{*}$ is a constant, i.e. it is independent of the choice of the point $p \in M_{1}$ and choice of the tangent vector $X$ in $k e r F_{*}$, then we say that $F$ is a slant submersion. In this case, the angle $\theta$ is called the slant angle of the slant submersion.

It is known that the distribution $k e r F_{*}$ is integrable. In fact, its leaves are $F^{-1}(q)$, $q \in M_{1}$, i.e., fibers. Thus it follows from above definition that the fibers are slant submanifolds of $M_{1}$, for slant submanifold, [11].

We first give some examples of slant submersions.

Example 7.1. Every Hermitian submersion from an almost Hermitian manifold onto an almost Hermitian manifold is a slant submersion with $\theta=0$.

Example 7.2. Every anti-invariant Riemannian submersion from an almost Hermitian manifold to a Riemannian manifold is a slant submersion with $\theta=\frac{\pi}{2}$.

A slant submersion is said to be proper if it is neither Hermitian nor anti-invariant Riemannian submersion.

Example 7.3. Let $F: R^{4} \longrightarrow R^{2}$ be a submersion defined by

$$
F\left(x_{1}, x_{2}, x_{3}, x_{4}\right)=\left(x_{1} \sin \alpha-x_{3} \cos \alpha, x_{4}\right) .
$$

Then for any $0<\alpha<\frac{\pi}{2}, F$ is a slant submersion with slant angle $\alpha$.

Example 7.4. The following Riemannian submersion defined by

$$
F\left(x_{1}, x_{2}, x_{3}, x_{4}\right)=\left(\frac{x_{1}-x_{4}}{\sqrt{2}}, x_{2}\right)
$$

is a slant submersion with slant angle $\theta=\frac{\pi}{4}$.

Let $F$ be a Riemannian submersion from an a Kähler manifold $\left(M_{1}, g_{1}, J\right)$ onto a Riemannian manifold $\left(M_{2}, g_{2}\right)$. Then for $X \in \Gamma\left(k e r F_{*}\right)$, we write

$$
J X=\phi X+\omega X
$$


where $\phi X$ and $\omega X$ are vertical and horizontal parts of $J X$. Also for $V \in \Gamma\left(\left(k e r F_{*}\right)^{\perp}\right)$, we have

$$
J Z=\mathcal{B} Z+\mathcal{C} Z,
$$

where $\mathcal{B Z}$ and $\mathcal{C} Z$ are vertical and horizontal components of $J Z$.

Using (5), (6), (28) and (30) we obtain

$$
\begin{aligned}
& \left(\nabla_{X} \omega\right) Y=\mathcal{C} \mathcal{T}_{X} Y-\mathcal{T}_{X} \phi Y \\
& \left(\nabla_{X} \phi\right) Y=\mathcal{B T}_{X} Y-\mathcal{T}_{X} \omega Y,
\end{aligned}
$$

where

$$
\begin{aligned}
& \left(\nabla_{X} \omega\right) Y=\mathcal{H} \nabla_{X} \omega Y-\omega \hat{\nabla}_{X} Y \\
& \left(\nabla_{X} \phi\right) Y=\hat{\nabla}_{X} \phi Y-\phi \hat{\nabla}_{X} Y .
\end{aligned}
$$

for $X, Y \in \Gamma\left(k e r F_{*}\right)$. Let $F$ be a slant submersion from an almost Hermitian manifold onto a Riemannian manifold, then we say that $\omega$ is parallel if $\nabla \omega=0$.

The proof of the following result is exactly same with slant immersions (see [11] or [8] for Sasakian case).

Theorem 7.1. [33]. Let $F$ be a Riemannian submersion from an almost Hermitian manifold $\left(M_{1}, g_{1}, J\right)$ onto a Riemannian manifold $\left(M_{2}, g_{2}\right)$. Then $F$ is a slant submersion if and only if there exists a constant $\lambda \in[-1,0]$ such that $\phi^{2} X=\lambda X$ for $X \in \Gamma\left(\right.$ ker $\left.F_{*}\right)$. Furthermore, if $F$ is a slant submersion, then $\lambda=-\cos ^{2} \theta$.

By using above theorem, it is easy to see that

$$
\begin{aligned}
& g_{1}(\phi X, \phi Y)=\cos ^{2} \theta g_{1}(X, Y) \\
& g_{1}(\omega X, \omega Y)=\sin ^{2} \theta g_{1}(X, Y)
\end{aligned}
$$

for any $X, Y \in \Gamma\left(k e r F_{*}\right)$.

The following theorem shows that the notion of slant submersions gives new information for the harmonicity of Riemannian submersions.

Theorem 7.2. [33]. Let $F$ be a slant submersion from a Kähler manifold onto a Riemannian manifold. If $\omega$ is parallel then $F$ is a harmonic map.

Slant submersions are also useful for obtaining new decomposition theorems.

Theorem 7.3. [33]. Let $F$ be a slant submersion from a Kähler manifold $\left(M_{1}, g_{1}, J_{1}\right)$ onto a Riemannian manifold $\left(M_{2}, g_{2}\right)$. Then $M_{1}$ is locally a product Riemannian manifold if and only if

$$
\begin{aligned}
g_{1}\left(\mathcal{H} \nabla_{Z_{1}} Z_{2}, \omega \phi X\right)= & g_{1}\left(\mathcal{A}_{Z_{1}} \mathcal{B} Z_{2}\right. \\
& \left.+\mathcal{H} \nabla_{Z_{1}} \mathcal{C} Z_{2}, \omega X\right)
\end{aligned}
$$


and

$$
\begin{aligned}
g_{1}\left(\mathcal{H} \nabla_{X} \omega \phi Y, Z_{1}\right)= & g_{1}\left(\mathcal{H} \nabla_{X} \omega Y, \mathcal{C} Z_{1}\right) \\
& +g_{1}\left(\mathcal{T}_{X} \omega Y, \mathcal{B} Z_{1}\right)
\end{aligned}
$$

for $X, Y, \in \Gamma\left(k e r F_{*}\right)$ and $Z_{1}, Z_{2} \in \Gamma\left(\left(k e r F_{*}\right)^{\perp}\right.$.

Finally, in this section, we give necessary and sufficient conditions for a slant submersion to be totally geodesic. We recall that a differentiable map $F$ between Riemannian manifolds $\left(M_{1}, g_{1}\right)$ and $\left(M_{2}, g_{2}\right)$ is called a totally geodesic map if $\left(\nabla F_{*}\right)(X, Y)=$ 0 for all $X, Y \in \Gamma\left(T M_{1}\right)$. A geometric interpretation of a totally geodesic map is that it maps every geodesic in the total manifold into a geodesic in the base manifold in proportion to arc lengths.

Theorem 7.4. Let $F$ be a slant submersion from a Kähler manifold $\left(M_{1}, g_{1}, J_{1}\right)$ onto a Riemannian manifold $\left(M_{2}, g_{2}\right)$. Then $F$ is totally geodesic if and only if

$$
\begin{aligned}
g_{1}\left(\mathcal{H} \nabla_{X} \omega \phi Y, Z_{1}\right)= & g_{1}\left(\mathcal{T}_{X} \omega Y, \mathcal{B} Z_{1}\right) \\
& +g_{1}\left(\mathcal{H} \nabla_{X} \omega Y, \mathcal{C} Z_{1}\right)
\end{aligned}
$$

and

$$
\begin{aligned}
-g_{1}\left(\mathcal{H} \nabla_{Z_{1}} \omega \phi X, Z_{2}\right)= & g_{1}\left(\mathcal{A}_{Z_{1}} B Z_{2}\right. \\
& \left.+\mathcal{H} \nabla_{Z_{1}} \mathcal{C} Z_{2}, \omega X\right)
\end{aligned}
$$

for $Z_{1}, Z_{2} \in \Gamma\left(\left(k e r F_{*}\right)^{\perp}\right)$ and $X, Y \in \Gamma\left(k e r F_{*}\right)$.

\section{Semi-invariant Submersions from Almost Hermitian Manifolds}

In this section, we define semi-invariant submersions, give examples, investigate the integrability of distributions and obtain a decomposition theorem by using the existence of such submersions.

Definition 8.1. [34]. Let $M_{1}$ be a complex $m$ - dimensional almost Hermitian manifold with Hermitian metric $g_{1}$ and almost complex structure $J$ and $M_{2}$ be a Riemannian manifold with Riemannian metric $g_{2}$. A Riemannian submersion $F$ : $M_{1} \longrightarrow M_{2}$ is called semi-invariant Riemannian submersion if there is a distribution $\mathcal{D}_{1} \subseteq k e r F_{*}$ such that

$$
\operatorname{ker} F_{*}=\mathcal{D}_{1} \oplus \mathcal{D}_{2}
$$

and

$$
J\left(\mathcal{D}_{1}\right)=\mathcal{D}_{1}, J\left(\mathcal{D}_{2}\right) \subseteq\left(k e r F_{*}\right)^{\perp},
$$


where $\mathcal{D}_{2}$ is orthogonal complementary to $\mathcal{D}_{1}$ in $\operatorname{ker} F_{*}$.

Above definition implies that the integral manifolds (fibers) $F^{-1}(q), q \in M_{2}$, of $\operatorname{ker} F_{*}$ is a CR-submanifold of $M_{1}$. For CR-submanifolds, see: [3], [10] and [38].

We now give some examples of semi-invariant Riemannian submersions.

Example 8.1. Every anti-invariant Riemannian submersion from an almost Hermitian manifold onto a Riemannian manifold is a semi-invariant Riemannian submersion with $\mathcal{D}_{1}=\{0\}$.

Example 8.2. Every holomorphic submersion from an almost Hermitian manifold onto an almost Hermitian manifold is a semi-invariant submersion with $\mathcal{D}_{2}=\{0\}$.

Example 8.3. Let $F: R^{6} \longrightarrow R^{3}$ be a submersion defined by $F\left(x_{1}, x_{2}, x_{3}, x_{4}\right.$, $\left.x_{5}, x_{6}\right)=\left(\frac{x_{1}+x_{2}}{\sqrt{2}}, \frac{x_{3}+x_{5}}{\sqrt{2}}, \frac{x_{4}+x_{6}}{\sqrt{2}}\right)$. Then it follows that $\operatorname{ker} F_{*}=\operatorname{span}\left\{V_{1}, V_{2}, V_{3}\right\}$ where

$$
\begin{gathered}
V_{1}=-\partial x_{1}+\partial x_{2}, V_{2}=-\partial x_{3}+\partial x_{5} \\
V_{3}=-\partial x_{4}+\partial x_{6} .
\end{gathered}
$$

On the other hand, we have $\left(k e r F_{*}\right)^{\perp}=\operatorname{span}\left\{X_{1}, X_{2}, X_{3}\right\}$, where

$$
\begin{gathered}
X_{1}=\partial x_{1}+\partial x_{2}, X_{2}=\partial x_{3}+\partial x_{5}, \\
\left.X_{3}=\partial x_{4}+\partial x_{6}\right\} .
\end{gathered}
$$

Hence we have $J V_{2}=V_{3}$ and $J V_{1}=-X_{1}$. Thus it follows that $\mathcal{D}_{1}=\operatorname{span}\left\{V_{2}, V_{3}\right\}$ and $\mathcal{D}_{2}=\operatorname{span}\left\{V_{1}\right\}$. Moreover one can see that $\mu=\operatorname{span}\left\{X_{2}, X_{3}\right\}$. By direct computations, we also have

$$
\begin{gathered}
g_{R^{6}}\left(J V_{1}, J V_{1}\right)=g_{R^{3}}\left(F_{*}\left(J V_{1}\right), F_{*}\left(J V_{1}\right)\right), \\
g_{R^{6}}\left(X_{2}, X_{2}\right)=g_{R^{3}}\left(F_{*}\left(X_{2}\right), F_{*}\left(X_{2}\right)\right)
\end{gathered}
$$

and

$$
g_{R^{6}}\left(X_{3}, X_{3}\right)=g_{R^{3}}\left(F_{*}\left(X_{3}\right), F_{*}\left(X_{3}\right)\right),
$$

which show that $F$ is a Riemannian submersion. Thus $F$ is a semi-invariant Riemannian submersion.

We now investigate the integrability of the distributions $\mathcal{D}_{1}$ and $\mathcal{D}_{2}$. Since fibers of semi-invariant submersions from Kähler manifolds are CR-submanifolds and $\mathcal{T}$ is the second fundamental form of the fibers, the following results can be deduced from Theorem 1.1 of [3].

Lemma 8.1. [34]. Let $F$ be a semi-invariant Riemannian submersion from a Kähler manifold $\left(M_{1}, g_{1}, J_{1}\right)$ onto a Riemannian manifold $\left(M_{2}, g_{2}\right)$. Then 
(i ) the distribution $\mathcal{D}_{2}$ is always integrable.

(ii) The distribution $\mathcal{D}_{1}$ is integrable if and only if

$$
g_{1}\left(T_{X} J Y-T_{Y} J X, J Z\right)=0
$$

for $X, Y \in \Gamma\left(\mathcal{D}_{1}\right)$ and $Z \in \Gamma\left(\mathcal{D}_{2}\right)$.

Let $F$ be a semi-invariant Riemannian submersion from a Kähler manifold $\left(M_{1}, g_{1}\right.$, $J)$ onto a Riemannian manifold $\left(M_{2}, g_{2}\right)$. We denote the complementary distribution to $J \mathcal{D}_{2}$ in $\left(k e r F_{*}\right)^{\perp}$ by $\mu$. Then for $V \in \Gamma\left(k e r F_{*}\right)$, we write

$$
J V=\phi V+\omega V,
$$

where $\phi V \in \Gamma\left(\mathcal{D}_{1}\right)$ and $\omega V \in \Gamma\left(J \mathcal{D}_{2}\right)$. Also for $X \in \Gamma\left(\left(k e r F_{*}\right)^{\perp}\right)$, we have

$$
J X=\mathcal{B} X+\mathcal{C} X
$$

where $\mathcal{B} X \in \Gamma\left(\mathcal{D}_{2}\right)$ and $\mathcal{C} X \in \Gamma(\mu)$.

Then, by using (36), (37), (5) and (6) we get

$$
\begin{aligned}
& \left(\nabla_{V} \phi\right) W=\mathcal{B T}_{V} W-\mathcal{T}_{V} \omega W \\
& \left(\nabla_{V} \omega\right) W=\mathcal{C} \mathcal{T}_{V} W-\mathcal{T}_{V} \phi W,
\end{aligned}
$$

for $V, W \in \Gamma\left(k e r F_{*}\right)$, where

$$
\left(\nabla_{V} \phi\right) W=\hat{\nabla}_{V} \phi W-\phi \hat{\nabla}_{V} W
$$

and

$$
\left(\nabla_{V} \omega\right) W=\mathcal{H} \nabla_{V}^{1} \omega W-\omega \hat{\nabla}_{V} W .
$$

The proof of the following proposition can be deduced from Theorem 5.1 of [3].

Proposition 8.1. [34]. Let $F$ be a semi-invariant Riemannian submersion from a Kähler manifold $\left(M_{1}, g_{1}, J\right)$ onto a Riemannian manifold $\left(M_{2}, g_{2}\right)$. Then the fibers of $F$ are locally product Riemannian manifolds if and only if $\left(\nabla_{V} \phi\right) W=0$ for $V, W \in \Gamma\left(k e r F_{*}\right)$.

The conditions for a semi-invariant submersion to be totally geodesic take the following forms.

Theorem 8.1. [34]. Let $F$ be a semi-invariant submersion from a Kähler manifold $\left(M_{1}, g_{1}, J\right)$ onto a Riemannian manifold $\left(M_{2}, g_{2}\right)$. Then $F$ is a totally geodesic map if and only if

(a) $\hat{\nabla}_{X} \phi Y+\mathcal{T}_{X} \omega Y$ and $\hat{\nabla}_{X} \mathcal{B} Z+\mathcal{T}_{X} \mathcal{C} Z$ belong to $\mathcal{D}_{1}$. 
(b) $\mathcal{H} \nabla_{X}^{1} \omega Y+T_{X} \phi Y$ and $\mathcal{T}_{X} \mathcal{B Z}+\mathcal{H} \nabla_{X}^{1} \mathcal{C} Z$ belong to $J \mathcal{D}_{2}$

for $X, Y \in \Gamma\left(k e r F_{*}\right)$ and $Z \in \Gamma\left(\left(k e r F_{*}\right)^{\perp}\right)$.

For the geometry of leaves of the distributions $\left(k e r F_{*}\right)^{\perp}$ and $k e r F_{*}$ we have the following results.

Proposition 8.2. [34]. Let $F$ be a semi-invariant submersion from a Kähler manifold $\left(M_{1}, g_{1}, J\right)$ onto a Riemannian manifold $\left(M_{2}, g_{2}\right)$. Then the distribution $\left(k e r F_{*}\right)^{\perp}$ defines a totally geodesic foliation if and only if

$$
\begin{gathered}
\mathcal{A}_{Z_{1}} \mathcal{B} Z_{2}+\mathcal{H} \nabla_{Z_{1}}^{1} \mathcal{C} Z_{2} \in \Gamma(\mu), \\
\mathcal{A}_{Z_{1}} \mathcal{C} Z_{2}+\mathcal{V} \nabla_{Z_{1}}^{1} Z_{2} \in \Gamma\left(\mathcal{D}_{2}\right)
\end{gathered}
$$

for $Z_{1}, Z_{2} \in \Gamma\left(\left(k e r F_{*}\right)^{\perp}\right)$.

Proposition 8.3. [34]. Let $F$ be a semi-invariant Riemannian submersion from a Kähler manifold $\left(M_{1}, g_{1}, J\right)$ onto a Riemannian manifold $\left(M_{2}, g_{2}\right)$. Then the distribution ker $F_{*}$ defines a totally geodesic foliation if and only if

$$
\begin{gathered}
\mathcal{T}_{X_{1}} \phi X_{2}+\mathcal{H} \nabla_{X_{1}}^{1} \omega X_{2} \in \Gamma\left(J \mathcal{D}_{2}\right), \\
\hat{\nabla}_{X_{1}} \phi X_{2}+\mathcal{T}_{X_{1}} \omega X_{2} \in \Gamma\left(\mathcal{D}_{1}\right)
\end{gathered}
$$

for $X_{1}, X_{2} \in \Gamma\left(\operatorname{ker} F_{*}\right)$.

We also have the following result.

Corollary 8.1. [34]. Let $F$ be a semi-invariant Riemannian submersion from a Kähler manifold $\left(M_{1}, g_{1}, J\right)$ onto a Riemannian manifold $\left(M_{2}, g_{2}\right)$. Then the distribution ker $F_{*}$ defines a totally geodesic foliation if and only if

$$
\begin{aligned}
g_{2}\left(\left(\nabla F_{*}\right)\left(X_{1}, X_{2}\right), F_{*}(J Z)\right) & =0, \\
g_{2}\left(\left(\nabla F_{*}\right)\left(X_{1}, \omega X_{2}\right), F_{*}(W)\right) & =-g_{1}\left(\mathcal{T}_{X_{1}} W, \phi X_{2}\right)
\end{aligned}
$$

for $X_{1}, X_{2} \in \Gamma\left(k e r F_{*}\right), Z \in \Gamma\left(\mathcal{D}_{2}\right)$. and $W \in \Gamma(\mu)$.

It is also possible to obtain the below decomposition theorem for semi-invariant submersions.

Theorem 8.3. [34]. Let $F$ be a semi-invariant submersion from a Kähler manifold $\left(M_{1}, g_{1}, J\right)$ onto a Riemannian manifold $\left(M_{2}, g_{2}\right)$. Then $M_{1}$ is locally a product Riemannian manifold $M_{\mathcal{D}_{1}} \times M_{\mathcal{D}_{2}} \times M_{\left(k e r F_{*}\right)} \perp$ if and only if

$$
(\nabla \phi)=0 \quad \text { on } \quad k e r F_{*},
$$




$$
\mathcal{A}_{Z_{1}} \mathcal{B} Z_{2}+\mathcal{H} \nabla_{Z_{1}}^{1} \mathcal{C} Z_{2} \in \Gamma(\mu)
$$

and

$$
\mathcal{A}_{Z_{1}} \mathcal{C} Z_{2}+\mathcal{V} \nabla_{Z_{1}}^{1} Z_{2} \in \Gamma\left(\mathcal{D}_{2}\right)
$$

for $Z_{1}, Z_{2} \in \Gamma\left(\left(k e r F_{*}\right)^{\perp}\right)$, where $M_{\mathcal{D}_{1}}, M_{\mathcal{D}_{2}}$ and $M_{\left(\text {ker } F_{*}\right)} \perp$ are integral manifolds of the distributions $\mathcal{D}_{1}, \mathcal{D}_{2}$ and $\left(k e r F_{*}\right)^{\perp}$.

Also by using above results, we have the following decomposition theorem.

Theorem 8.4. [34]. Let $F$ be a semi-invariant submersion from a Kähler manifold $\left(M_{1}, g_{1}, J\right)$ onto a Riemannian manifold $\left(M_{2}, g_{2}\right)$. Then $M_{1}$ is locally a product Riemannian manifold $M_{\text {ker } F_{*}} \times M_{\left(k e r F_{*}\right) \perp}$ if and only if

$$
\begin{aligned}
& g_{2}\left(\left(\nabla F_{*}\right)\left(X_{1}, X_{2}\right), F_{*}(J Z)\right)=0, \\
& g_{2}\left(\left(\nabla F_{*}\right)\left(X_{1}, \omega X_{2}\right), F_{*}(W)\right)=-g_{1}\left(\mathcal{T}_{X_{1}} W, \phi X_{2}\right), \\
& \mathcal{A}_{Z_{1}} \mathcal{B} Z_{2}+\mathcal{H} \nabla_{Z_{1}}^{1} \mathcal{C} Z_{2} \in \Gamma(\mu), \\
& \mathcal{A}_{Z_{1}} \mathcal{C} Z_{2}+\mathcal{V} \nabla_{Z_{1}}^{1} Z_{2} \in \Gamma\left(\mathcal{D}_{2}\right)
\end{aligned}
$$

for $X_{1}, X_{2} \in \Gamma\left(k e r F_{*}\right), W \in \Gamma(\mu), Z \in \Gamma\left(\mathcal{D}_{2}\right)$ and $Z_{1}, Z_{2} \in \Gamma\left(\left(k e r F_{*}\right)^{\perp}\right)$, where $M_{\text {ker } F_{*}}$ and $M_{\left(k e r F_{*}\right)} \perp$ are integral manifolds of the distributions ker $F_{*}$ and $\left(k e r F_{*}\right)^{\perp}$.

In the rest of this section, we will obtain a classification theorem for semi-invariant submersions from Kähler manifolds. Let $F$ be a Riemannian submersion from a Riemannian manifold onto a Riemannian manifold $\left(M_{2}, g_{2}\right)$. Recall that a Riemannian submersion is called a Riemannian submersion with totally umbilical fibers if

$$
\mathcal{T}_{X} Y=g_{1}(X, Y) H
$$

for $X, Y \in \Gamma\left(\operatorname{ker} F_{*}\right)$, where $H$ is the mean curvature vector field of the fiber. We also recall that a simply connected complete Kähler manifold of constant sectional curvature $c$ is called a complex space-form, denoted by $M(c)$. The curvature tensor of $M(c)$ is

$$
\begin{aligned}
R(X, Y) Z= & \frac{c}{4}[g(Y, Z) X-g(X, Z) Y \\
& +g(J Y, Z) J X-g(J X, Z) J Y \\
& +2 g(X, J Y) J Z]
\end{aligned}
$$

for $X, Y, Z \in \Gamma(T M)$. Moreover, from [24] we have the following relation for a Riemannian submersion

$$
\begin{aligned}
g_{1}\left(R^{1}\left(X_{1}, X_{2}\right) X_{3}, Z\right)= & g_{1}\left(\left(\nabla_{X_{2}} \mathcal{T}\right)_{X_{1}} X_{3}, Z\right) \\
& -g_{1}\left(\left(\nabla_{X_{1}} \mathcal{T}\right)_{X_{2}} X_{3}, Z\right)
\end{aligned}
$$


for $X_{1}, X_{2}, X_{3} \in \Gamma\left(k e r F_{*}\right)$ and $Z \in \Gamma\left(\left(k e r F_{*}\right)^{\perp}\right)$, where $R^{1}$ is the curvature tensor field of $M_{1}$ and $(\nabla \mathcal{T})$ is the covariant derivative of $\mathcal{T}$.

By using (40) and (41), as in CR-submanifolds, see: Theorem 1.2 of [3], we have the following result.

Teorem 8.5. Let $F$ be a semi-invariant submersion with totally umbilical fibers from a complex space form $\left(M_{1}(c), g_{1}, J\right)$ onto a Riemannian manifold $\left(M_{2}, g_{2}\right)$. Then $c=0$.

We now give a classification theorem for semi-invariant Riemannian submersions with totally umbilical fibers. But we need the following result which shows that the mean curvature vector field of semi-invariant Riemannian submersions has special form.

Lemma 8.2. [34]. Let $F$ be a semi-invariant submersion with totally umbilical fibers from a Kähler manifold $\left(M_{1}, g_{1}, J\right)$ onto a Riemannian manifold $\left(M_{2}, g_{2}\right)$. Then $H \in \Gamma\left(J \mathcal{D}_{2}\right)$.

We now give a classification theorem for a semi-invariant submersion with totally umbilical fibers which is similar to that Theorem 6.1 of [38].

Theorem 8.6. [34]. Let $F$ be a semi-invariant submersion with totally umbilical fibers from a Kähler manifold $\left(M_{1}, g_{1}, J\right)$ onto a Riemannian manifold $\left(M_{2}, g_{2}\right)$. Then either $\mathcal{D}_{2}$ is one dimensional or the fibers are totally geodesic.

\section{Semi-Slant Submersions from Almost Hermitian Manifolds}

In this section, we define semi-slant submersions, give examples, investigate the integrability of distributions and give necessary and sufficient conditions for such submersions to be totally geodesic.

Definition 9.1. [29]. Let $M_{1}$ be a complex $m$ - dimensional almost Hermitian manifold with Hermitian metric $g_{1}$ and almost complex structure $J$ and $M_{2}$ be a Riemannian manifold with Riemannian metric $g_{2}$. A Riemannian submersion $F$ : $M_{1} \longrightarrow M_{2}$ is called semi-slant Riemannian submersion if there is a distribution $\mathcal{D}_{1} \subseteq \operatorname{ker} F_{*}$ such that

$$
\operatorname{ker} F_{*}=\mathcal{D}_{1} \oplus \mathcal{D}_{2}, J\left(\mathcal{D}_{1}\right)=\mathcal{D}_{1}
$$

and the angle $\theta(X)$ between $J X$ and the space $\left(\mathcal{D}_{2}\right)_{q}$ is constant for non-zero $X \in$ $\left(\mathcal{D}_{2}\right)_{q}$ and $q \in M_{1}$. 
We note that above definition implies that the integral manifold (fiber) $F^{-1}(q)$, $q \in M_{2}$, of $k e r F_{*}$ is a semi-slant-submanifold of $M_{1}$. For semi-slant submanifolds, see: [26].

We now give some examples of semi-slant Riemannian submersions.

Example 9.1. Every slant Riemannian submersion from an almost Hermitian manifold onto a Riemannian manifold is a semi-slant Riemannian submersion with $\mathcal{D}_{1}=\{0\}$.

Example 9.2. Every semi-invariant submersion from an almost Hermitian manifold onto an almost Hermitian manifold is a semi-invariant submersion with $\theta=\frac{\pi}{2}$.

We say that a semi-slant submersion is proper if $\mathcal{D}_{1} \neq\{0\}$ and $\theta \neq 0, \frac{\pi}{2}$. Here is an example for proper semi-slant submersions.

Example 9.3. [29]. Let $F: R^{10} \longrightarrow R^{4}$ be a submersion defined by

$$
F\left(x_{1}, \ldots, x_{10}\right)=\left(\frac{x_{3}-x_{5}}{\sqrt{2}}, x_{6}, \frac{x_{7}-x_{9}}{\sqrt{2}}, x_{8}\right) .
$$

Then it follows that $F$ is a semi-slant submersion such that

$$
\mathcal{D}_{1}=S p\left\{V_{1}=\partial x_{1}, V_{2}=\partial x_{2}\right\}
$$

and

$$
\mathcal{D}_{2}=S p\left\{V_{3}=\partial x_{3}+\partial x_{5}, V_{4}=\partial x_{7}+\partial x_{9}, V_{5}=\partial x_{4}, V_{6}=\partial x_{10}\right\}
$$

with the slant angle $\frac{\pi}{4}$.

Let $F$ be a semi-slant Riemannian submersion from a Kähler manifold $\left(M_{1}, g_{1}, J\right)$ onto a Riemannian manifold $\left(M_{2}, g_{2}\right)$. Then for $V \in \Gamma\left(k e r F_{*}\right)$, we write

$$
J V=\phi V+\omega V
$$

where $\phi V \in \Gamma\left(k e r F_{*}\right)$ and $\omega V \in \Gamma\left(\left(k e r F_{*}\right)^{\perp}\right)$. Also for $X \in \Gamma\left(\left(k e r F_{*}\right)^{\perp}\right)$, we have

$$
J X=\mathcal{B} X+\mathcal{C} X
$$

where $\mathcal{B} X \in \Gamma\left(k e r F_{*}\right)$ and $\mathcal{C} X \in \Gamma\left(\left(k e r F_{*}\right)^{\perp}\right)$. We denote the complementary distribution to $\omega \mathcal{D}_{2}$ in $\left(k e r F_{*}\right)^{\perp}$ by $\mu$. Then we have

$$
\left(k e r F_{*}\right)^{\perp}=\omega \mathcal{D}_{2} \oplus \mu .
$$

For the integrability of the distributions we have the following results. 
Theorem 9.1. [29]. Let $F$ be a semi-slant submersion from an almost Hermitian manifold $\left(M_{1}, g_{1}, J\right)$ onto a Riemannian manifold $\left(M_{2}, g_{2}\right)$. Then the complex distribution $D_{1}$ is integrable if and only if, for $X, Y \in \Gamma\left(\mathcal{D}_{1}\right)$, we have

$$
\omega\left(\hat{\nabla}_{X} Y-\hat{\nabla}_{Y} X\right)=\mathcal{C}\left(\mathcal{T}_{X} Y-\mathcal{T}_{Y} X\right) .
$$

Theorem 9.2. [29]. Let $F$ be a semi-slant submersion from an almost Hermitian manifold $\left(M_{1}, g_{1}, J\right)$ onto a Riemannian manifold $\left(M_{2}, g_{2}\right)$. Then the slant distribution $\mathcal{D}_{2}$ is integrable if and only if, for $X, Y \in \Gamma\left(\mathcal{D}_{2}\right)$, we have

$$
P\left(\hat{\nabla}_{X} \phi Y-\hat{\nabla}_{Y} \phi X+\mathcal{T}_{X} \omega Y-\mathcal{T}_{Y} \omega X\right)=0,
$$

where $P$ denotes the projection on the distribution $k e r F_{*}$.

For the geometry of leaves of the vertical and horizontal distributions we have the following theorems.

Theorem 9.3. [29]. Let $F$ be a semi-slant submersion from an almost Hermitian manifold $\left(M_{1}, g_{1}, J\right)$ onto a Riemannian manifold $\left(M_{2}, g_{2}\right)$. Then the vertical distribution ker $F_{*}$ defines totally geodesic foliation on $M_{1}$ if and only if, for $X, Y \in \Gamma\left(k e r F_{*}\right)$, we have

$$
\omega\left(\hat{\nabla}_{X} \phi Y+\mathcal{T}_{X} \omega Y\right)+\mathcal{C}\left(\mathcal{T}_{X} \phi Y+\mathcal{H} \nabla_{Y} \omega Y\right)=0 .
$$

Theorem 9.4. [29]. Let $F$ be a semi-slant submersion from an almost Hermitian manifold $\left(M_{1}, g_{1}, J\right)$ onto a Riemannian manifold $\left(M_{2}, g_{2}\right)$. Then the vertical distribution $k e r F_{*}$ defines totally geodesic foliation on $M_{1}$ if and only if, for $X, Y \in \Gamma\left(\left(k e r F_{*}\right)^{\perp}\right)$, we have

$$
\phi\left(\mathcal{V} \nabla_{X} B Y+\mathcal{A}_{X} \mathcal{C} Y\right)+\mathcal{B}\left(\mathcal{A}_{X} \mathcal{B} Y+\mathcal{H} \nabla_{X} \mathcal{C} Y\right)=0 .
$$

We also have the following result which gives necessary and sufficient conditions for a semi-slant submersion to be totally geodesic.

Theorem 9.5. [29]. Let $F$ be a semi-slant submersion from an almost Hermitian manifold $\left(M_{1}, g_{1}, J\right)$ onto a Riemannian manifold $\left(M_{2}, g_{2}\right)$. Then $F$ is totally geodesic map if and only if we have

$$
\omega\left(\hat{\nabla}_{X} Y+\mathcal{T} X \omega Y\right)+\mathcal{C}\left(\mathcal{T}_{X} \phi Y+\mathcal{H} \nabla_{X} \omega Y\right)=0
$$

and

$$
\omega\left(\nabla_{X} \mathcal{B Z}+\mathcal{T}_{X} \mathcal{C} Z\right)+\mathcal{C}\left(\mathcal{T}_{X} \mathcal{B} Z+\mathcal{H} \nabla_{X} \mathcal{C} Z\right)=0
$$

for $X, Y \in \Gamma\left(\left(k e r F_{*}\right)\right.$ and $Z \in \Gamma\left(\left(k e r F_{*}\right)^{\perp}\right)$.

Also for the harmonicity of semi-slant submersion we have the following. 
Theorem 9.6. [29]. Let $F$ be a semi-slant submersion from an almost Hermitian manifold $\left(M_{1}, g_{1}, J\right)$ onto a Riemannian manifold $\left(M_{2}, g_{2}\right)$. Then $F$ is harmonic if and only if

$$
\operatorname{trace}\left(\nabla F_{*}\right)=0 \quad \text { on } \quad \mathcal{D}_{2} .
$$

We also note that Park and Prasad obtained more results on the geometry of semislant submersions in [29]. In particular, they found curvature relations among the base manifold, the total manifold and fibers.

\section{Open Problems}

In this section, we propose some open problems to the interested readers.

(1) It is known that the sectional curvature in the base manifold increases by the amount $\frac{3}{4} g_{M}\left([X, Y]^{\mathcal{V}},[X, Y]^{\mathcal{V}}\right)$. Although, in [29], the authors investigated curvature relations for semi-slant submersions, the relations for anti-invariant submersions, slant submersions and semi-invariant submersions has not been investigated yet. So it would be interesting to research curvature relations for such submersions.

(2) Oniciuc[25] proved that Riemannian submersion $F: M_{1} \rightarrow M_{2}$ is biharmonic if and only if $\nabla^{M} \tau(F)=0$, where $M_{2}$ is compact, orientable and $R i c^{M_{2}} \leq 0$ and $\tau(F)$ denotes the tension field of $F$. So it would be interesting to find necessary and sufficient conditions for anti-invariant submersions, slant submersions, semiinvariant submersions and semi-slant submersions to be biharmonic.

(3) Some special vector fields play important roles in Riemannian geometry, for instance Killing vector fields. A smooth vector field $X$ on a Riemannian manifold $M$ is said to be Killing if its local flow consists of local isometries of the Riemannian manifold $(M, g)$. The presence of a nonzero Killing vector field on a compact Riemannian manifold constrains its geometry as well as topology. For Riemannian submersions, it is known that if the tension field $\tau(F)$ is a unitary Killing vector field then $F$ is biharmonic [25]. On the other hand, holomorphic vector fields are useful in complex geometry. Note that a complex vector field $Z$ of type $(1,0)$ on $M$ is said to be holomorphic vector field if $Z f$ is holomorphic for locally defined holomorphic function $f$. Therefore one can investigate the effect of such vector fields ( especially for the harmonicity or biharmonicity of such maps) on the geometry of anti-invariant submersions, slant submersions. Similarly, one can check the effect of infinitesimal automorphism (analytical vector field) of an almost complex structure on the biharmonicity of anti-invariant submersions, slant submersions, semi-invariant submersions and semi-slant submersions.

(4) It is known that every Hermitian manifold is even dimensional. On the other hand, almost contact metric manifolds are odd dimensional. And their geometry 
is different from the geometry of Hermitian manifolds. The contact version of holomorphic submersions have been studied by many authors under the name of almost contact submersions, see [12] and [15]. So it will be interesting problem to research anti-invariant submersions, slant submersions, semi-invariant submersions and semi-slant submersions from almost contact metric manifolds onto Riemannian manifolds. We note that Park studied same problem for quaternion Kähler manifolds, [27], [28].

(5) It is known that a Riemannian submersion $F$ is harmonic if and only if its fibers are minimal. The harmonicity conditions of anti-invariant submersions, slant submersions and semi-slant submersions have been given in Theorem 6.5, Theorem 7.2 and Theorem 9.6. So next problem is to determine stability of such submersions. Here we note that any harmonic map from a compact Riemannian manifold to a manifold of non-positive sectional curvatures is stable, [2].

(6) Some special differential or partial differential equations defined on Riemannian manifolds are useful for obtaining decomposition theorems. One can also argue the existence of a domain manifold for a differential equation or a partial differential equation to possess a nontrivial solution. Recently, in [16], the authors introduce a (global) partial differential equation on a Riemannian manifold, called the local Möbius equation, and in the case of a solution to this equation, they showed that manifold locally decomposes to certain products of Riemannian manifolds. For instance, if $F:\left(M_{1}, g_{1}\right) \rightarrow\left(M_{2}, g_{2}\right)$ is a submersion and satisfies the Möbius equation, then they showed that $\left(M_{1}, g_{1}\right)$ is locally twisted product manifold. So it would be possible to obtain nice results by assuming anti-invariant submersions, slant submersions, semi-invariant submersions and semi-slant submersions satisfy some special equations on the total manifold. Conversely, one can also investigate the following problem: are there special differential equations or partial differential equations on Hermitian manifolds such that anti-invariant submersions, slant submersions, semi-invariant submersions or semi-slant submersions are solutions of such equations.

(7) As a generalization of slant submanifolds and semi-slant submanifolds, antislant submanifolds were introduced by Carriazo[9] and such submanifolds were studied in detail in [31] under the name of hemi-slant submanifolds. Then one can define and study hemi-slant submersions as generalization of slant submersions and semi-slant submersions. One can see that the fibers of such submersions will be hemi-slant submanifolds.

\section{REFERENCES}

1. C. Altafini, Redundant robotic chains on Riemannian submersions, IEEE Transactions on Robotics and Automation, 20(2) (2004), 335-340. 
2. P. Baird and J. C. Wood, Harmonic Morphisms Between Riemannian Manifolds, London Mathematical Society Monographs, No. 29, Oxford University Press, The Clarendon Press, Oxford, 2003.

3. A. Bejancu, Geometry of CR-submanifolds, Kluwer Academic, 1986.

4. R. Bhattacharyaa and V. Patrangenarub, Nonparametic estimation of location and dispersion on Riemannian manifolds, Journal of Statistical Planning and Inference, 108 (2002), 23-35.

5. R. L. Bishop and B. O'Neill, Manifolds of negative curvature, Trans. Amer. Math. Soc, 145 (1969), 1-49.

6. J. P. Bourguignon, A mathematician's visit to Kaluza-Klein theory, Rend. Sem. Mat. Univ. Poi. Torino Fascicolo Speciale 1989, P. D. E. and Geometry, 1988.

7. F. Bullo and A. D. Lewis, Geometric Control of Mechanical Systems, Springer-Verlag, 2004.

8. J. L. Cabrerizo, A. Carriazo, L. M. Fernandez and M. Fernandez, Semi-slant Submanifolds of a Sasakian Manifold, Geometriae Dedicata, 78 (1999), 183-1999.

9. A. Carriazo, Bi-slant immersions, in: Proc. ICRAMS, 2000, Kharagpur, India, (2000), 88-97.

10. B. Y. Chen, Riemannian submanifolds, in: Handbook of Differential Geometry, (E. Dillen and L. Verstraelen, eds.) Vol I, 2000, pp. 187-418.

11. B. Y. Chen, Geometry of slant submanifolds, Katholieke Universiteit Leuven, 1990.

12. D. Chinea, Almost contact metric submersions, Rend. Circ. Mat. Palermo, 34(1) (1985), 89-104.

13. G. De Rham, Sur la reducibilite dun espace de Riemann, Comment. Math. Helv, 26 (1952), 328-344.

14. M. Falcitelli, S. Ianus, A. M. Pastore and M. Visinescu, Some applications of Riemannian submersions in physics, Romanian Journal of Physics, 48(4-5) (2003).

15. M. Falcitelli, S. Ianus and A. M. Pastore, Riemannian Submersions and Related Topics, World Scientific, River Edge, NJ, 2004.

16. M. Fernandez-Lopez, E. Garcia-Rio and D. N. Kupeli, The local Möbius equation and decomposition theorems in Riemannian geometry, Canad. Math. Bull., 45(3) (2002), 378-387.

17. A. Gray, Pseudo-Riemannian almost product manifolds and submersions, J. Math. Mech., 16 (1967), 715-737.

18. S. W. Hawking and G. F. R. Ellis, The Large Scale Structure of Space-time, Cambridge University Press, 1973.

19. S. Hiepko, Eine innere Kennzeichnung der verzerrten produkte, Math. Ann., 241 (1979), 209-215. 
20. S. Ianus, R. Mazzocco and G. E. Vilcu, Riemannian submersions from quaternionic manifolds, Acta Appl. Math., 104(1) (2008), 83-89.

21. L. C. Kennedy, Some Results on Einstein metrics on two classes of quotient manifolds, $\mathrm{PhD}$ thesis, University of California, 2003.

22. J. C. Marrero and J. Rocha, Locally conformal Kähler submersions, Geom. Dedicata, 52(3) (1994), 271-289.

23. F. Memoli, G. Sapiro and P. Thompson, Implicit brain imaging, Neuro Image, 23 (2004), 179-188.

24. B. O'Neill, The fundamental equations of a submersion, Mich. Math. J., 13 (1966), 458-469.

25. C. Oniciuc, Biharmonic maps between Riemannian manifolds, An. Stiint. Univ. Al. I. Cuza Iaşi. Mat. (N.S.), 48 (2002), 237-248.

26. N. Papaghiuc, Semi-slant submanifolds of a Kählerian manifold, An. Ştiint. Univ. Al. I. Cuza Iaşi. Sect. I a Mat., 40(1) (1994), 55-61.

27. K. S. Park, H-slant submersions, Bull. Korean Math. Soc., 49(2) (2012), 329-338.

28. K. S. Park, H-semi-invariant submersions, Taiwanese Journal of Mathematics, 16(5) (2012), 1865-1878.

29. K. S. Park and R. Prasad, Semi-slant submersions, Bull. Korean Math. Soc., in press.

30. R. Ponge and H. Reckziegel, Twisted products in pseudo Riemannian geometry, Geom. Dedic., 48 (1993), 15-25.

31. B. Sahin, Warped product submanifolds of Kaehler manifolds with a slant factor, Ann. Polon. Math., 95(3) (2009), 207-226.

32. B. Sahin, Anti-invariant Riemannian submersions from almost Hermitian manifolds, Central European J. Math., 8(3) (2010), 437-447.

33. B. Sahin, Slant submersions from almost Hermitian manifolds, Bull. Math. Soc. Sci. Math. Roumanie Tome, 54(102) No. 1, (2011), 93-105.

34. B. Sahin, Semi-invariant Riemannian submersions from almost Hermitian manifolds, Canadian Mathematical Bulletin, doi:10.4153/CMB-2011-144-8, to appear.

35. M. W. Spong, S. Hutchinson and M. Vidyasagar, Robot Modeling and Control, Wiley, 2005.

36. M. Wang and W. Ziller, Einstein metrics on principal torus bundles, J. Diff. Geo., 31 (1990), 215-248.

37. B. Watson, Almost Hermitian submersions, J. Differential Geometry, 11(1) (1976), 147165.

38. K. Yano and M. Kon, Structures on Manifolds, World Scientific, Singapore, 1984.

39. H. Zhao, A. R. Kelly, J. Zhou, J. Lu and Y. Y. Yang, Graph attribute embedding via Riemannian submersion learning, Computer Vision and Image Understanding, 115 (2011), 962-975. 


\section{Bayram Şahin}

Inonu University

Department of Mathematics

44280 Malatya

Turkey

E-mail: bayram.sahin@inonu.edu.tr 